\title{
A NEW SIMPLE PROOF OF THE AZTEC DIAMOND THEOREM
}

\author{
MANUEL FENDLER AND DANIEL GRIESER
}

\begin{abstract}
The Aztec diamond of order $n$ is the union of lattice squares in the plane intersecting the square $|x|+|y|<n$. The Aztec diamond theorem states that the number of domino tilings of this shape is $2^{n(n+1) / 2}$. It was first proved by Elkies, Kuperberg, Larsen and Propp in 1992. We give a new simple proof of this theorem.
\end{abstract}

A domino is a $1 \times 2$ rectangle in the plane whose corners are lattice points, i.e. have integer coordinates. A domino tiling of a subset $S$ of the plane is a covering of $S$ by a set of dominoes whose interiors are disjoint. The problem of counting the number of domino tilings (or tilings, for short) of a given set $S$ has received much attention in the last 50 years, partly because of its significance in physics, but also because of the many beautiful mathematical structures that have appeared in its study. For example, the number of domino tilings of a $2 \times n$ rectangle is the $n$th Fibonacci number, and there is a rather non-trivial formula for the number of domino tilings of an $m \times n$-rectangle due to Kasteleyn [5], Fisher and Temperley [9], which for even $m=n$ reads $2^{n^{2} / 2} \prod_{j, k=1}^{n / 2}\left(\cos ^{2} \frac{j \pi}{n+1}+\cos ^{2} \frac{k \pi}{n+1}\right)$. One of the amazing facts in this area is that when essentially rotating the square by 45 degrees the number of tilings is given by a much simpler formula.

More precisely, define the Aztec diamond of order $n$ by

$$
A_{n}=\bigcup\{Q: Q \cap\{(x, y):|x|+|y|<n\} \neq \emptyset\}
$$

where $Q$ ranges over squares $[k, k+1] \times[l, l+1]$ with $k, l \in \mathbb{Z}$. See Figure 1

Theorem 1 (2]). Denote by $T_{n}$ the number of domino tilings of $A_{n}$. Then

$$
T_{n}=2^{n(n+1) / 2} .
$$

See also Remark 4 for a refinement. The first four proofs of this formula were given in [2], 3], later other proofs appeared in 1], 4] (these two proofs are essentially identical) and [7. See [6] and references given there for a more refined discussion of domino tilings, and 8 for a recent generalization of the theorem.

In this note we give a proof of this theorem which is inspired by the first proof in 2, but simplifies it in various respects, for example we replace the height function arguments used there by a very simple direct construction, see Lemma 2 .

Date: July 8, 2018.

2010 Mathematics Subject Classification. 05A15.

Key words and phrases. domino tilings. 


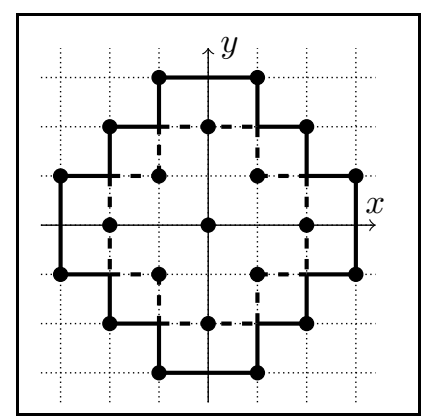

Figure 1. The Aztec Diamonds of orders 2 (dashed lines) and 3 (heavy lines). The marked points are nodes $(n=2)$.

We use induction on $n$. Clearly $T_{1}=2$, so in order to prove the theorem it suffices to show that

$$
T_{n+1}=2^{n+1} T_{n} .
$$

We need a few preparations before we give the proof of this recursion. We fix $n$ throughout. The strategy is to associate a 'field of arrows' to each tiling, and then to relate the fields of arrows arising from $A_{n}$ tilings to those arising from $A_{n+1}$ tilings.

Nodes and lattice squares. Consider the two Aztec diamonds $A_{n} \subset A_{n+1}$. We call a lattice point $(i, j) \in A_{n+1}$ satisfying $i+j \equiv n \bmod 2$ a node. In particular, the extreme points of $A_{n+1}$ are nodes. All other nodes are contained in $A_{n}$ and are called interior nodes. See Figure 1 for the case $n=2$. A lattice square will be a $1 \times 1$ square contained in $A_{n+1}$ whose corners are lattice points. A boundary square is a lattice square contained in $A_{n+1}$ but not in $A_{n}$. Notice that each lattice square has exactly two corners which are nodes, and each interior node is adjacent to four lattice squares.

Fields of arrows. Suppose in each lattice square of $A_{n+1}$ we draw an arrow pointing from one corner node to the other. We call this collection of arrows a field of arrows if it satisfies the following condition.

Arrow field condition: Each interior node $N$ is either:

- attracting: all adjacent arrows point towards $N$, or

- repelling: all adjacent arrows point away from $N$, or

- transient: any two collinear arrows adjacent to $N$ point in the same direction.

See Figure 2 for the six possible local arrow patterns. We call a field of arrows pointing outward/inward if all arrows in boundary squares point outward/inward, see Figure 3 (B) and (C) for examples.

From tilings to fields of arrows. We now associate fields of arrows to tilings. More precisely, we define an outward (resp. inward) pointing field of arrows $F(\mathcal{T})$ on $A_{n+1}$ for each tiling $\mathcal{T}$ of $A_{n+1}$ (resp. $A_{n}$ ). This is done domino by domino by the rule indicated in Figure 3 (A) 1$]$

\footnotetext{
${ }^{1}$ Note that the field of arrows is always defined on all of $A_{n+1}$, even for a tiling of $A_{n}$.
} 


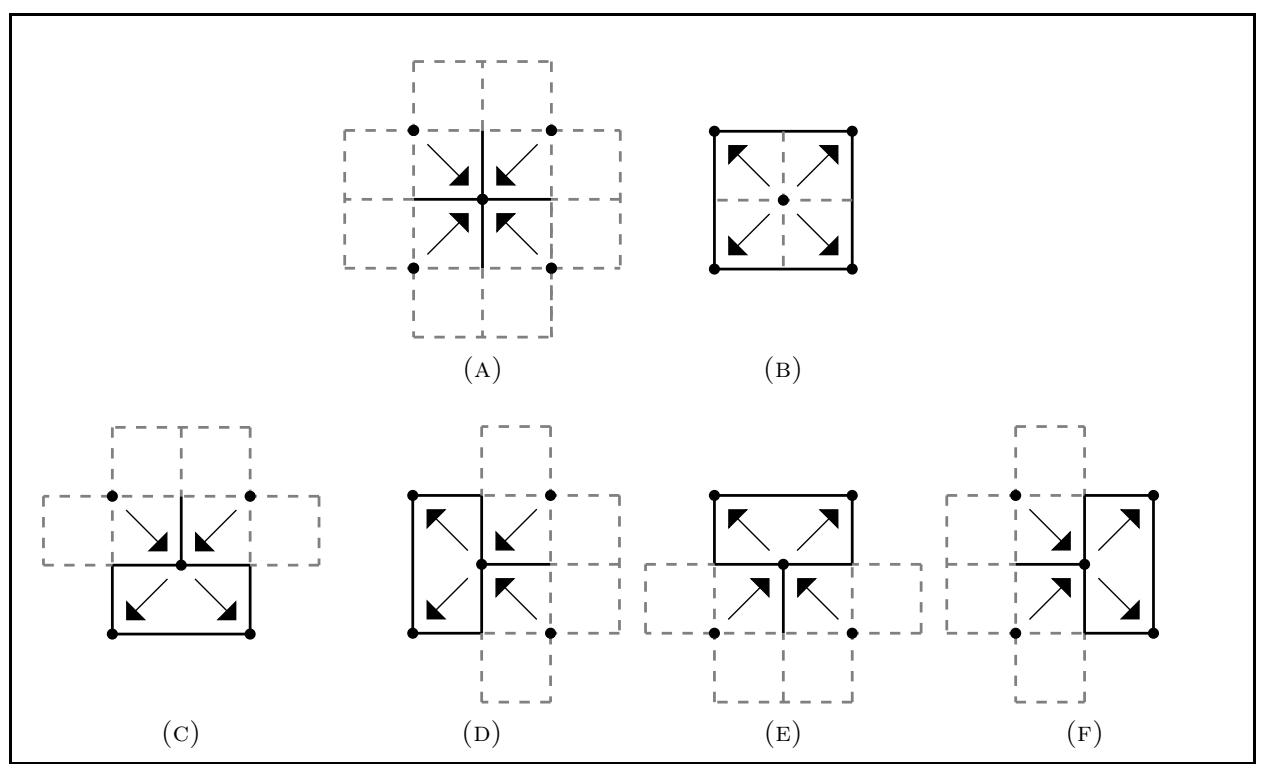

Figure 2. The six possible local arrow patterns, and how they arise from domino tilings. The precise position of the dominoes on dashed lines is inessential.

More explicitly, consider first a tiling $\mathcal{T}$ of $A_{n+1}$. Define $F(\mathcal{T})$ as follows: Each domino in the tiling has exactly two corners which are nodes. The two arrows contained in that domino are chosen to point towards these corner nodes. Since each boundary domino has a corner node lying on the boundary of $A_{n+1}$, all arrows in boundary squares point outward. Also, by looking at the ways that dominoes can lie adjacent to any interior node, we see that the arrow field condition is satisfied. See Figure 2, and Figure 3 (B) for an example.

For a tiling $\mathcal{T}$ of $A_{n}$ define $F(\mathcal{T})$ by putting arrows in the lattice squares of $A_{n}$ by the same rule as for $A_{n+1}$, and in addition putting inward pointing arrows in all boundary squares of $A_{n+1}$. Then the arrow field condition is satisfied for the same reason as before, since the arrows in boundary squares may be thought of as arising from horizontal dominoes added along the outside boundary of $A_{n}$.

The core of the argument is the proof of the following lemma. Denote by $r(\mathcal{F})$ the number of repelling nodes for a field of arrows $\mathcal{F}$.

Lemma 2 (Number of tilings for a fixed field of arrows). Given an inward (resp. outward) pointing field of arrows $\mathcal{F}$, there are precisely $2^{r(\mathcal{F})}$ domino tilings $\mathcal{T}$ of $A_{n}$ (resp. $A_{n+1}$ ) satisfying $F(\mathcal{T})=\mathcal{F}$.

In particular, every outward (resp. inward) pointing field of arrows arises from some tiling of $A_{n+1}$ (resp. $A_{n}$ ).

Proof. First consider an outward pointing field of arrows $\mathcal{F}$. For each lattice square, draw the two sides of the square which lie in the direction of its arrow in bold face, see Figure 4. Note that if $\mathcal{F}$ comes from a tiling then these bold face lines must be boundary lines of dominoes. 


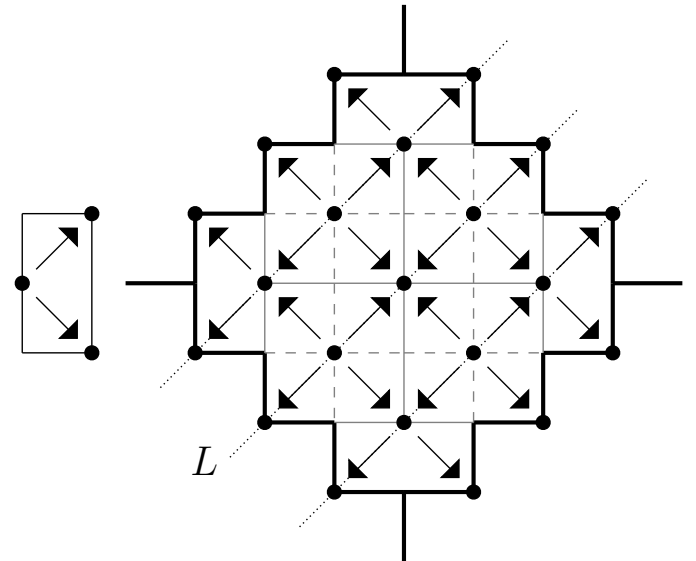

(A)

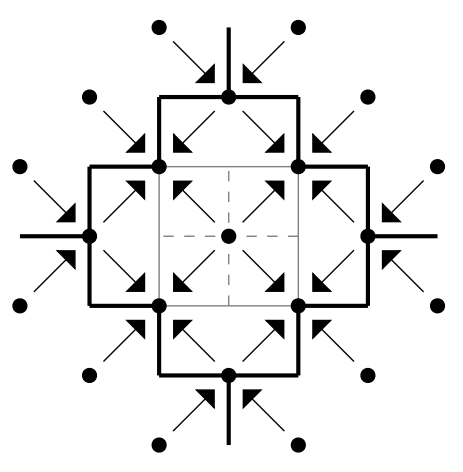

(c)

Figure 3. (A) A single domino with nodes and arrows; (B) resp. (C) Outward resp. inward pointing field of arrows $(n=2)$, and tilings of $A_{n+1}$ resp. $A_{n}$ from which they arise. In the $2 \times 2$ squares containing a dashed cross, the position of dominoes can be either horizontal or vertical.

The union $\mathcal{B}$ of all these bold lines divides $A_{n+1}$ into components (the connected components of $\left.A_{n+1} \backslash \mathcal{B}\right)$.

Claim: Each component is either a $1 \times 2$ rectangle or a $2 \times 2$ square, where the latter are precisely those $2 \times 2$ squares having repelling nodes at their center.

Proof of Claim: Consider a lattice square $S$. W.l.o.g. assume that the lower left corner $P$ and the upper right corner $Q$ of $S$ are nodes, and that the arrow of $S$ points towards $P$. Since $\mathcal{F}$ is outward pointing, the node $Q$ must be an interior node. It can be either repelling or transient. If it is repelling then the component containing $S$ is the $2 \times 2$ square centered at $Q$ (Figure 4(A)). If it is transient then there are two possibilities for the arrows adjacent to $Q$, and in both cases the component containing $S$ is a $1 \times 2$ rectangle (Figure $4(\mathrm{~B})$ and $(\mathrm{C})$ ). This proves the claim.

The claim implies that $\mathcal{F}$ determines the tilings $\mathcal{T}$ having $F(\mathcal{T})=\mathcal{F}$, except for the choice of having two vertical or two horizontal dominoes in each $2 \times 2$ square. Conversely, any tiling that fits into the decomposition will have $\mathcal{F}$ as field of arrows. Since the number of $2 \times 2$ squares is $r(\mathcal{F})$, the lemma is proved in the case of outward pointing $\mathcal{F}$.

Now consider an inward pointing field of arrows $\mathcal{F}$. Define $\mathcal{B}$ as above. Since $\mathcal{F}$ is inward pointing, the boundary of $A_{n}$ is a subset of $\mathcal{B}$. Now the same argument as above, only looking at components contained in $A_{n}$, shows that there are precisely $2^{r(\mathcal{F})}$ tilings of $A_{n}$ having $\mathcal{F}$ as field of arrows.

The other ingredient in the proof is the following lemma. 


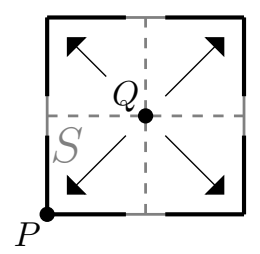

(A)

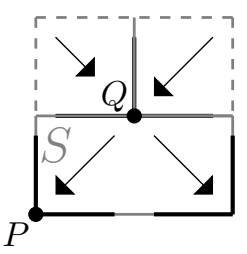

(B)

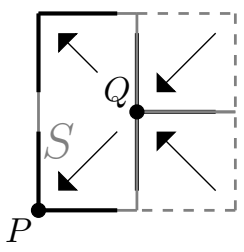

(C)

Figure 4. Determining components from fields of arrows, Lemma 2

Lemma 3. For any outward pointing field of arrows on $A_{n+1}$ let $r$ be the number of repelling nodes and a the number of attracting nodes. Then

$$
r-a=n+1 .
$$

Proof. The interior nodes of $A_{n+1}$ lie on $n+1$ lines running south-west to northeast, and on each line there are $n+2$ arrows. See Figure 3(B).

We claim that on each line there is one more repellent node than there are attracting nodes. Since there are $n+1$ lines, this will imply the lemma.

To prove the claim, fix one line $L$ and consider the sequence of arrows on $L$ and their changes of direction when traversing $L$ from south-west to north-east. Each arrow points forward (f) or backward (b). Changes b-f happens precisely at repelling nodes, and changes $\mathrm{f}-\mathrm{b}$ at attracting nodes. There is no change of direction at transient nodes. Since the first arrow points $b$ and the last arrow points $f$, there must be one more change b-f than $\mathrm{f}-\mathrm{b}$, which was to be shown.

Proof of the recursion (1). Let $\mathcal{O}$ (resp. $\mathcal{I}$ ) be the sets of outward (inward) pointing fields of arrows on $A_{n+1}$. Reversing the direction of each arrow preserves the arrow field condition and therefore defines the map

$$
\text { flip : } \mathcal{O} \rightarrow \mathcal{I}
$$

which is its own inverse, hence bijective. For any $\mathcal{F} \in \mathcal{O}$, the attracting nodes of $\mathcal{F}$ are the repelling nodes of flip $(\mathcal{F})$, hence Lemma 3 implies that $r(\mathcal{F})=r(f l i p(\mathcal{F}))+$ $n+1$. Lemma 2 then implies that the number of tilings of $A_{n+1}$ corresponding to $\mathcal{F}$ equals $2^{n+1}$ times the number of tilings of $A_{n}$ corresponding to flip $(\mathcal{F})$. Summing over all $\mathcal{F} \in \mathcal{O}$ yields (1).

Figures 3 (B) and (C) show an example of an outward pointing field of arrows and its flip.

Remark 4. The flip defined in (2) is the same map as the domino shuffling map defined in the fourth proof in [3], although the definition of that map is different and requires proof of well-definedness, which is obvious in our setting 2 Also, as shown there, it is straight-forward to prove a refinement of Theorem 1]: The number of domino tilings of $A_{n}$ having exactly $2 k$ horizontal dominoes is

$$
\left(\begin{array}{c}
\frac{n(n+1)}{2} \\
k
\end{array}\right)
$$

\footnotetext{
${ }^{2}$ In this correspondence, the nodes in this paper correspond to odd vertices in 3 .
} 
for $k=0, \ldots, \frac{n(n+1)}{2}$. The central observation in the inductive proof of this fact is the following: The numbers of horizontal $1 \times 2$ components corresponding to a

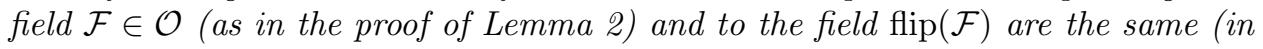
Figure $3(B)$ and $(C)$ there are two such components). This is obvious from Figure 母 $(B)$ and $(C)$ since the flip rotates these patterns by 180 degrees.

\section{REFERENCES}

[1] Richard A. Brualdi and Stephen Kirkland. Aztec diamonds and digraphs, and Hankel determinants of Schröder numbers. J. Combin. Theory Ser. B, 94(2):334-351, 2005.

[2] Noam Elkies, Greg Kuperberg, Michael Larsen, and James Propp. Alternating-sign matrices and domino tilings. I. J. Algebraic Combin., 1(2):111-132, 1992.

[3] Noam Elkies, Greg Kuperberg, Michael Larsen, and James Propp. Alternating-sign matrices and domino tilings. II. J. Algebraic Combin., 1(3):219-234, 1992.

[4] Sen-Peng Eu and Tung-Shan Fu. A simple proof of the Aztec diamond theorem. Electron. J. Combin., 12:Research Paper 18, 8 pp. (electronic), 2005.

[5] P.W. Kasteleyn. The statistics of dimers on a lattice: I. The number of dimer arrangements on a quadratic lattice. Physica, 27(12):1209 - 1225, 1961.

[6] Richard Kenyon and Andrei Okounkov. What is ... a dimer? Notices Amer. Math. Soc., 52(3):342-343, 2005.

[7] K. Kokhas'. Domino tilings of Aztec diamonds and squares. Zap. Nauchn. Sem. S.-Peterburg. Otdel. Mat. Inst. Steklov. (POMI), 360(Teoriya Predstavlenii, Dinamicheskie Sitemy, Kombinatornye Metody. XVI):180-230, 298, 2008.

[8] Tri Lai. A generalization of Aztec diamond theorem, part I. Electron. J. Combin., 21(1):Paper $1.51,19,2014$.

[9] H. N. V. Temperley and Michael E. Fisher. Dimer problem in statistical mechanics-an exact result. Philos. Mag. (8), 6:1061-1063, 1961.

Institut für Mathematik, Carl von Ossietzky Universität Oldenburg, 26111 OldenBURG, Germany

E-mail address: manuel.fendler@uni-oldenburg.de

Institut für Mathematik, Carl von Ossietzky Universität Oldenburg, 26111 OldenBURG, GERMANY

E-mail address: daniel.grieser@uni-oldenburg.de 